\title{
SCDM-k: Localized orbitals for solids via selected columns of the density matrix
}

\author{
Anil Damle ${ }^{\mathrm{a}, *}$, Lin Lin ${ }^{\mathrm{a}, \mathrm{b}}$, Lexing Ying ${ }^{\mathrm{d}, \mathrm{c}}$ \\ ${ }^{a}$ Department of Mathematics, University of California, Berkeley, Berkeley, CA 94720 \\ ${ }^{b}$ Computational Research Division, Lawrence Berkeley National Laboratory, Berkeley, CA \\ 94720 \\ ${ }^{c}$ Institute for Computational and Mathematical Engineering, Stanford University, Stanford, \\ CA 94305 \\ ${ }^{d}$ Department of Mathematics, Stanford University, Stanford, CA 94305
}

\begin{abstract}
The recently developed selected columns of the density matrix (SCDM) method [J. Chem. Theory Comput. 11, 1463, 2015] is a simple, robust, efficient and highly parallelizable method for constructing localized orbitals from a set of delocalized Kohn-Sham orbitals for insulators and semiconductors with $\Gamma$ point sampling of the Brillouin zone. In this work we generalize the SCDM method to Kohn-Sham density functional theory calculations with k-point sampling of the Brillouin zone, which is needed for more general electronic structure calculations for solids. We demonstrate that our new method, called SCDM-k, is by construction gauge independent and a natural way to describe localized orbitals. SCDM-k computes localized orbitals without the use of an optimization procedure, and thus does not suffer from the possibility of being trapped in a local minimum. Furthermore, the computational complexity of using SCDM-k to construct orthogonal and localized orbitals scales as $\mathcal{O}(N \log N)$ where $N$ is the total number of $\mathbf{k}$-points in the Brillouin zone. SCDM-k is therefore efficient even when a large number of $\mathbf{k}$-points are used for Brillouin zone sampling. We demonstrate the numerical performance of SCDM-k using systems with model potentials in two and three dimensions.
\end{abstract}

Keywords: Kohn-Sham density functional theory, Localized orbitals, Brillouin zone sampling, Density matrix, Interpolative decomposition

\section{Introduction}

Kohn-Sham density functional theory (DFT) $[1,2]$ is the most widely used electronic structure theory for molecules and systems in condensed phase. The

\footnotetext{
* Corresponding author

Email addresses: damle@berkeley.edu (Anil Damle), linlin@math.berkeley.edu (Lin Lin), lexing@math.stanford.edu (Lexing Ying)
}

Preprint submitted to Journal of Computational Physics

October 4, 2016

(C) 2016. This manuscript version is made available under the Elsevier user license http://www.elsevier.com/open-access/userlicense/1.0/ 
Kohn-Sham orbitals (a.k.a. Kohn-Sham wavefunctions) are eigenfunctions of the Kohn-Sham Hamiltonian. We refer to the span of a given set of Kohn-Sham orbitals as the Kohn-Sham invariant subspace. These orbitals are in general delocalized, i.e. each orbital has significant magnitude across the entire computational domain. However, information about atomic structure and chemical bonding, which is often localized in real space, may be difficult to interpret from delocalized Kohn-Sham orbitals. The connection between localized and delocalized information is made possible by a localization procedure.

A localization procedure finds a set of orbitals that are localized in real space, and span the Kohn-Sham invariant subspace. Examples of widely used localization schemes include Boys localization [3] mostly in the context of chemistry, and maximally localized Wannier functions (MLWFs) $[4,5]$ mostly in the context of physics and materials science. The localized orbitals are not only useful for analyzing chemical and materials systems, but can also serve as powerful computational tools for hybrid functional calculations [6,7], theory of polarization of crystalline solids based on Berry-phase calculations [8], interpolation of band structure [4], linear scaling DFT calculations [9], and excited state theories $[10,11]$ among others. Because of the wide range of applications for localized orbitals, several other localization methods have also been proposed in the past few years $[12-16]$.

The potential for constructing localized orbitals from delocalized Kohn-Sham orbitals can be justified physically by the "nearsightedness" principle for electronic matter of finite HOMO-LUMO gap [17, 18]. The nearsightedness principle can be more rigorously stated as the single particle density matrix (DM) being exponentially localized along the off-diagonal direction in its real space representation [19, 17, 20-23]. Based on the exponential decay of the DM in the real space, we have recently developed the selected columns of the density matrix (SCDM) method [16] as a new way to construct localized orbitals. The method is simple, robust, efficient and highly parallelizable. As the name suggests, the localized orbitals are obtained directly from a column selection procedure implicitly applied to the density matrix. Hence, the locality of these columns is a direct consequence of the locality of the density matrix. In contrast with Boys localized orbitals or MLWFs, our method does not attempt to minimize a given localization measure via a minimization procedure. Consequently, our method does not require any initial guess of localized orbitals, and its cost is predetermined for a given problem size. It also avoids some of the potential problems associated with a minimization scheme, such as getting stuck at a local minimum.

For isolated molecules, the number of electrons is relatively small. On the other hand, the number of electrons in solids can reach macroscopic scale, and the calculation must be simplified. Using the fact that the potential and the electron density are periodic with respect to the unit cell of a solid system, one can perform a Bloch decomposition of the Kohn-Sham Hamiltonian. The wavefunctions for each Bloch decomposed Hamiltonian satisfy twisted boundary conditions indexed by a vector $\mathbf{k}$ belonging to the so-called Brillouin zone. In order to compute physical quantities such as the electron density and total 
energy in Kohn-Sham DFT, the Brillouin zone needs to be represented by a number of discrete k-points. This is called Brillouin zone sampling. We refer readers to section 3 as well as [24] for more details of the Bloch decomposition and Brillouin zone sampling. In particular, the scheme using one special $\mathbf{k}$ point, denoted by $\Gamma=(0,0,0)^{T}$, to sample the Brillouin zone is called the $\Gamma$ point sampling scheme. The SCDM procedure proposed in Ref. [16] is applicable to Kohn-Sham DFT calculations for isolated molecules, and for solids with $\Gamma$ point sampling of the Brillouin zone.

In many physics and materials science applications such as chemical bonding analysis of complex solids, band structure interpolation, and Berry-phase theories, localized orbitals need to be constructed from Kohn-Sham orbitals obtained from a set of $\mathbf{k}$-points in the Brillouin zone other than the $\Gamma$ point. The number of $\mathbf{k}$ points needed is system dependent, and can range from tens to tens of thousands. The common practice for Brillouin zone sampling is to diagonalize the Kohn-Sham Hamiltonian matrix for each k-point independently. Since the Kohn-Sham Hamiltonian matrix is in general complex Hermitian, the Kohn-Sham orbitals obtained for each $\mathbf{k}$-point can acquire an arbitrary phase, often referred to as the "gauge" of the orbitals. For degenerate orbitals (i.e. orbitals with the same eigenvalue) the gauge can be an arbitrary unitary matrix. The widely used method for finding MLWFs [5] is gauge-dependent. It involves the differentiation operator with respect to the Brillouin zone index k. Therefore a gauge transformation needs to be performed prior to the minimization procedure to smooth the gauge, so that the differentiation operator is well defined [4]. Such a gauge smoothing procedure is not unique. After the gauge transformation, the computation of MLWFs requires the minimization of a nonlinear, non-convex energy functional. Therefore, the iterative procedure may get stuck at local minimum. Furthermore, the nonlinear energy functional and its solution can depend heavily on the initial guess. This is especially the case for materials where within the unit cell there is complex atomic structure.

In this paper, we generalize the SCDM procedure for finding localized orbitals to solids with k-point sampling. The new method, which we refer to as SCDM-k, has a few notable features. First, the localized orbitals are obtained directly from columns of the density matrix, which is a gauge invariant quantity. Thus, SCDM-k does not require a gauge transformation, and the result is independent of the choice of the gauge. Second, SCDM-k is a direct method that does not involve an iterative optimization procedure and thus avoids getting stuck at a local minimum. Third, SCDM-k has only one parameter to adjust (size of the local supercell), which is introduced to improve the efficiency, and our numerical experiments indicate that the quality of the localized orbitals is relatively insensitive to the choice of this parameter. Finally, the SCDM-k procedure is highly efficient. The complexity for generating non-orthogonal and orthogonal localized orbitals is $\mathcal{O}(N)$ and $\mathcal{O}(N \log N)$, respectively where $N$ is the total number of $\mathbf{k}$-points in the Brillouin zone. Therefore the method is suitable even when a large number of $\mathbf{k}$-points are used for sampling the Brillouin zone.

The paper is organized as follows. Section 2 outlines the notation and some 
concepts that will be used throughout this paper. Section 3 then outlines the procedure for solving Kohn-Sham DFT with Brillouin zone sampling. After briefly reviewing the SCDM procedure for the $\Gamma$ point case, we describe in section 4 the SCDM-k procedure for k-point sampling. Finally, section 5 presents numerical results in two and three dimensions using a model potential and is followed by concluding remarks and future directions in section 6 .

\section{Preliminaries}

\subsection{Notation}

A relatively self-contained discussion of $\mathbf{k}$-point sampling requires the introduction of a considerable amount of notation. Table 1 summarizes the requisite notation that we will be using throughout this manuscript. We also provide a brief overview of some of the more pervasive notation used throughout the rest of the text, and introduce the remainder as needed. In the discussion below, without loss of generality we assume the dimension $d=3$, and the formulation can be easily extended to $d=1$ or $d=2$.

We denote a unit cell by $\Omega^{u}$. The global supercell, denoted by $\Omega^{g}$, contains $N_{1} \times N_{2} \times N_{3}$ unit cells equipped with periodic boundary conditions. Due to the translational symmetry, the problem on a global supercell can be equivalently decomposed into $N_{1} \times N_{2} \times N_{3}$ independent problems on a unit cell, each represented by a k-point in the Brillouin zone using a Monkhorst-Pack grid [25]. One important component of the SCDM-k method is the so-called local supercell $\Omega^{\ell}$ associated with the unit cell $\Omega^{u}$. A local supercell is comprised of $N_{1}^{\ell} \times N_{2}^{\ell} \times N_{3}^{\ell}$ adjacent unit cells $\left(N_{i}^{\ell} \leq N_{i}, i=1,2,3\right)$. Figure 1 illustrates the described relationship between the unit cell, local supercell, and global supercell in a two dimensional setting.

Computationally, each k-point problem can be solved with any suitable discrete basis set. Below we assume a planewave basis set is used with $M_{1} \times M_{2} \times M_{3}$ grid points in reciprocal space. This corresponds to a set of grid points of the same size in real space discretizing $\Omega^{u}$ uniformly. With a slight abuse of notation, this set of discrete grid points in the unit cell is also denoted by $\Omega^{u}$. A similar abuse of notation is used for the global supercell $\Omega^{g}$ and the local supercell $\Omega^{\ell}$.

To present the algorithms generally, we allow for distinct numbers of points in each of the three dimensions. This is the case for both the real space grid of the unite cell, and the k-point grid. However, often the asymptotic computational cost will only be a function of the product of the number of points per dimension. Therefore, we use capital letters with subscripts such as $N_{1}, N_{2}, N_{3}$ to define the number of points per dimension and the same capital letter sans subscript such as $N$ to denote the total number of points. In addition, we use calligraphic letters such as $\mathcal{K}$ to denote sets. These will be used for operations such as general indexing of matrices or summation. 


\begin{tabular}{|c|c|}
\hline Notation & Description \\
\hline$\Omega^{u}$ & $\begin{array}{l}\text { Unit cell; Collection of indices corresponding to uni- } \\
\text { form real space grid points in the unit cell }\end{array}$ \\
\hline$\Omega^{\ell}$ & $\begin{array}{l}\text { Local supercell; Collection of indices corresponding } \\
\text { to uniform real space grid points in a local supercell }\end{array}$ \\
\hline$\Omega^{g}$ & $\begin{array}{l}\text { Global supercell; Collection of indices corresponding } \\
\text { to uniform real space grid points in a global supercell }\end{array}$ \\
\hline$\imath$ & Imaginary unit $\sqrt{-1}$ \\
\hline $\mathbf{e}_{1}, \mathbf{e}_{2}, \mathbf{e}_{3}$ & Unit vector along each dimension \\
\hline $\mathbf{s}=\left(s_{1}, s_{2}, s_{3}\right)$ & Shift vector of the Monkhorst-Pack grid \\
\hline $\mathbf{k}=\left(k_{1}, k_{2}, k_{3}\right)$ & A k-point \\
\hline$M_{1}, M_{2}, M_{3}$ & $\begin{array}{l}\text { Number of uniform grid points in the unit cell along } \\
\text { each dimension }\end{array}$ \\
\hline$N_{1}, N_{2}, N_{3}$ & $\begin{array}{l}\text { Number of k-points for Brillouin zone sampling along } \\
\text { each dimension }\end{array}$ \\
\hline$N_{1}^{\ell}, N_{2}^{\ell}, N_{3}^{\ell}$ & $\begin{array}{l}\text { Number of unit cells in a local supercell along each } \\
\text { dimension }\end{array}$ \\
\hline$L_{1}, L_{2}, L_{3}$ & Length of the unit cell $\Omega^{u}$ along each dimension \\
\hline$M$ & $M_{1} \times M_{2} \times M_{3}$ \\
\hline$N$ & $N_{1} \times N_{2} \times N_{3}$ \\
\hline$N^{\ell}$ & $N_{1}^{\ell} \times N_{2}^{\ell} \times N_{3}^{\ell}$ \\
\hline $\mathcal{K}$ & $\begin{array}{l}\text { Collection of all the k-points of the Monkhorst-Pack } \\
\text { grid corresponding to the global supercell }\end{array}$ \\
\hline $\mathcal{K}^{\ell}$ & $\begin{array}{l}\text { Collection of all the k-points of the Monkhorst-Pack } \\
\text { grid corresponding to a local supercell }\end{array}$ \\
\hline$n_{b}$ & Number of wavefunctions in the unit cell \\
\hline$\psi_{b, \mathbf{k}}$ & $\begin{array}{l}\text { A Kohn-Sham orbital on the global supercell } \Omega^{g} \text {, } \\
\text { both at the continuous and the discrete level }\end{array}$ \\
\hline$\psi_{b, \mathbf{k}}^{\ell}$ & $\begin{array}{l}\text { A Kohn-Sham orbital on the local supercell } \Omega^{\ell} \text {, both } \\
\text { at the continuous and the discrete level }\end{array}$ \\
\hline$u_{b, \mathbf{k}}$ & $\begin{array}{l}\text { Periodic part of a Kohn-Sham orbital on the unit cell } \\
\Omega^{u} \text {, both at the continuous and the discrete level }\end{array}$ \\
\hline$P_{\mathbf{k}}$ & $\begin{array}{l}\text { Density matrix corresponding a particular } \mathbf{k} \text { point } \\
\text { on the global supercell } \Omega^{g} \text { at the discrete level }\end{array}$ \\
\hline$P$ & $\begin{array}{l}\text { Total density matrix on the global supercell } \Omega^{g} \text { at } \\
\text { the discrete level }\end{array}$ \\
\hline $\mathcal{C}$ & $\begin{array}{l}\text { Collection of indices for the selected columns on the } \\
\text { unit cell } \Omega^{u}\end{array}$ \\
\hline $\mathcal{C}^{g}$ & $\begin{array}{l}\text { Collection of all indices for the selected columns on } \\
\text { the global supercell } \Omega^{g}\end{array}$ \\
\hline
\end{tabular}

Table 1: Notation used in the paper 


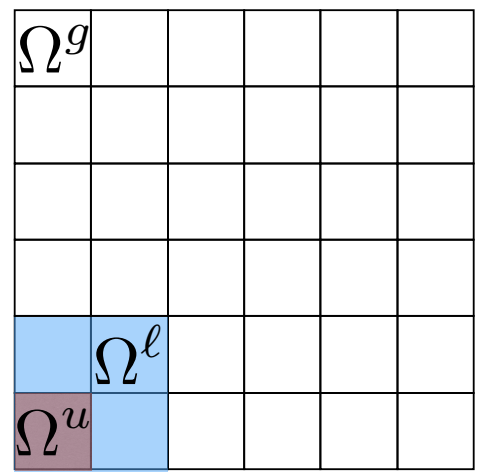

Figure 1: Illustration of the relationship in two dimension $(d=2)$ between the unit cell (shaded red), local supercell (shaded blue), and global supercell corresponding to $N_{1}=N_{2}=6$ and $N_{1}^{\ell}=N_{2}^{\ell}=2$.

\subsection{Sub-selection of matrices}

Because we deal with very large matrices that exhibit structure due to the underlying problem set up, it is very useful for us to associate quantities from the physical problem with portions of matrices. Therefore, we use set subscripts to denote sub-selection of rows and columns of matrices. For example, $A_{\{1,2\},\{3,4\}}$ is a submatrix of $A$ consisting of the intersection of rows one and two with columns three and four. We use ":" as a subscript to denote that all rows or columns are considered, i.e. $A_{:, 1}$ denotes the first column of $A$.

\subsection{Column-pivoted $Q R$ factorizations}

Because they play a central role in the development of our algorithms, we briefly introduce column-pivoted factorizations. Consider $A \in \mathbb{R}^{n_{b} \times M}$, where the sizes have been chosen to coincide with the matrices we will actually be performing these factorizations on later. A QR factorization with column pivoting (QRCP) of $A$ follows the algorithm in [26] to compute an $M \times M$ permutation matrix $\Pi$, a $n_{b} \times n_{b}$ orthogonal matrix $Q$, a $n_{b} \times n_{b}$ upper triangular matrix $R$ and a $n_{b} \times\left(M-n_{b}\right)$ matrix $T$ such that

$$
A \Pi=Q\left[\begin{array}{ll}
R & T
\end{array}\right] .
$$

The column pivoting algorithm in [26] is a greedy procedure to try and ensure that $R$ is as well conditioned as possible and that its singular values do not differ too much from those of $A$. If we let $\mathcal{C}$ denote the original indices of the $n_{b}$ columns permuted to the front by $\Pi$ then we observe that $A_{:, \mathcal{C}}=Q R$. Hence, if $R$ is well conditioned we expect these columns to form a well conditioned basis for the range of $A$. Finally, What we have presented here is a very narrow definition of such factorizations, and we direct the reader to [27] and [28] for a more through treatment of such algorithms. 


\section{Kohn-Sham density functional theory with Brillouin zone sampling}

In this section we provide a relatively self-contained description of KohnSham DFT, and focus particularly on Brillouin zone sampling due to the periodic structure. A more detailed discussion can be found in, e.g., [24].

\subsection{Continuous formulation}

For a crystalline solid modeled by a global supercell $\Omega^{g}$ consisting of $N$ unit cells with each unit cell containing $2 n_{b}$ electrons (the factor of two comes from spin), the Kohn-Sham equations are [2]

$$
H \psi_{\alpha}(\mathbf{r})=-\frac{1}{2} \nabla^{2} \psi_{\alpha}(\mathbf{r})+V(\mathbf{r}) \psi_{\alpha}(\mathbf{r})=\varepsilon_{\alpha} \psi_{\alpha}(\mathbf{r}), \quad \mathbf{r} \in \Omega^{g}, \quad \alpha=1, \ldots, n_{b} N
$$

Each eigenfunction $\psi_{\alpha}$ satisfies the Born-von Karman (BvK) boundary condition, which is the periodic boundary condition on $\Omega^{g}$

$$
\psi_{\alpha}\left(\mathbf{r}+N_{i} L_{i} \mathbf{e}_{i}\right)=\psi_{\alpha}(\mathbf{r}), \quad \forall \mathbf{r} \in \Omega^{g}, \quad i=1,2,3 .
$$

Using the $\mathrm{BvK}$ boundary condition, all eigenvalues $\left\{\varepsilon_{\alpha}\right\}$ are real, and all eigenfunctions $\left\{\psi_{\alpha}\right\}$ are orthogonal to each other under the $L^{2}$ inner product. We assume the eigenvalues are ordered in a non-descending manner.

Kohn-Sham DFT requires solving for the $n_{b} N$ eigenfunctions associated with the lowest eigenvalues $\left\{\varepsilon_{\alpha}\right\}_{\alpha=1}^{n_{b} N}$. $\varepsilon_{\alpha}$ is called a Kohn-Sham orbital energy, and $\psi_{\alpha}$ is called a Kohn-Sham orbital or a Kohn-Sham wavefunction. In Kohn-Sham DFT, $V(\mathbf{r})$ is the self-consistent single particle potential, and self-consistency is usually reached through an iterative procedure. Here without loss of generality we assume self-consistency has been reached. We also assume a pseudopotential is used so $V(\mathbf{r})$ is smooth and can be discretized using uniform grid points. Nonlocal pseudopotentials are neglected for simplicity of notation, and do not introduce any extra numerical difficulty when added. We refer readers to [24] for more detailed explanation of the terminology.

For crystalline solids, $V(\mathbf{r})$ is a periodic function in $\Omega^{u}$, i.e.

$$
V\left(\mathbf{r}+L_{i} \mathbf{e}_{i}\right)=V(\mathbf{r}), \quad \forall \mathbf{r} \in \Omega^{g}, \quad i=1,2,3 .
$$

The Bloch theory (or Bloch-Floquet theory) states that the $n_{b} N$ Kohn-Sham wavefunctions can be relabeled using two indices $\alpha=(b, \mathbf{k})$, so that $\psi_{\alpha} \equiv \psi_{b, \mathbf{k}}$ can be decomposed into the form

$$
\psi_{b, \mathbf{k}}(\mathbf{r})=e^{\imath \mathbf{k} \cdot \mathbf{r}} u_{b, \mathbf{k}}(\mathbf{r}),
$$

where $u_{b, \mathbf{k}}(\mathbf{r})$ is the periodic part of $\psi_{b, \mathbf{k}}(\mathbf{r})$ satisfying

$$
u_{b, \mathbf{k}}\left(\mathbf{r}+L_{i} \mathbf{e}_{i}\right)=u_{b, \mathbf{k}}(\mathbf{r}), \quad \forall \mathbf{r} \in \Omega^{g}, \quad i=1,2,3 .
$$

Using the Bloch decomposition, Eq. (1) can be written in terms of $u$ on each unit cell $\Omega^{u}$ as

$$
-\frac{1}{2}(\nabla+i \mathbf{k})^{2} u_{b, \mathbf{k}}(\mathbf{r})+V(\mathbf{r}) u_{b, \mathbf{k}}(\mathbf{r})=\varepsilon_{b, \mathbf{k}} u_{b, \mathbf{k}}(\mathbf{r}), \quad \mathbf{r} \in \Omega^{u},
$$


Where each $\mathbf{k}$ is a point in the first Brillouin zone defined as

$$
\mathcal{B}=\left[-\frac{\pi}{L_{1}}, \frac{\pi}{L_{1}}\right] \times\left[-\frac{\pi}{L_{2}}, \frac{\pi}{L_{2}}\right] \times\left[-\frac{\pi}{L_{3}}, \frac{\pi}{L_{3}}\right] .
$$

For each k-point, $b=1, \ldots, n_{b}$, i.e. $n_{b}$ is the number of wavefunctions per kpoint. For simplicity we will drop the $b$ subscript when describing properties that hold for each $u_{b, \mathbf{k}}(\mathbf{r}), b=1, \ldots, n_{b}$. There are a few $\mathbf{k}$-points in the Brillouin zone that play special roles in crystallography and also in numerical computation. The most important one is the $\Gamma$ point, which is the origin of the Brillouin zone.

In order to solve Eq. (6), the Brillouin zone $\mathcal{B}$ needs to be discretized. One of the most widely used discretization schemes is the so-called Monkhorst-Pack grid [25], which uses a uniform discretization of $\mathcal{B}$. The discretized set of $\mathbf{k}$ points is

$$
\mathcal{K}=\left\{\left(\frac{2 \pi j_{1}}{N_{1} L_{1}}, \frac{2 \pi j_{2}}{N_{2} L_{2}}, \frac{2 \pi j_{3}}{N_{3} L_{3}}\right)+\mathbf{s} \mid j_{i}=-\frac{N_{i}}{2}+1, \ldots, \frac{N_{i}}{2}, \quad i=1,2,3\right\},
$$

where $\mathbf{s}$ is a shift vector, and we assume $N_{i}$ is an even number. Two common choices are $\mathbf{s}=(0,0,0)$ (no shift) and $\mathbf{s}=\left(\frac{\pi}{N_{1} L_{1}}, \frac{\pi}{N_{2} L_{2}}, \frac{\pi}{N_{3} L_{3}}\right)$ (half grid shift). It should be noted that the inclusion of a non-zero shift vector could violate the BvK boundary condition. But this only adds an optional post-processing procedure for handling the phase vector and will be discussed in section 4.5. For now on we assume $\mathbf{s}=(0,0,0)$, and the BvK boundary condition holds because

$\psi_{b, \mathbf{k}}\left(\mathbf{r}+N_{i} L_{i} \mathbf{e}_{i}\right)=e^{\imath \mathbf{k} \cdot\left(\mathbf{r}+N_{i} L_{i} \mathbf{e}_{i}\right)} u_{b, \mathbf{k}}\left(\mathbf{r}+N_{i} L_{i} \mathbf{e}_{i}\right)=e^{\imath \mathbf{k} \cdot N_{i} L_{i} \mathbf{e}_{i}} \psi_{b, \mathbf{k}}(\mathbf{r})=\psi_{b, \mathbf{k}}(\mathbf{r})$.

The last equality holds because

$$
e^{\imath \mathbf{k} \cdot N_{i} L_{i} \mathbf{e}_{i}}=1,
$$

which is satisfied for $\mathbf{k} \in \mathcal{K}$.

\subsection{Discrete formulation}

For each $\mathbf{k} \in \mathcal{K}$, Eq. (6) is solved numerically for $b=1, \ldots, n_{b}$, and we assume the resulting eigenfunctions are solved for and represented on a uniform grid discretizing the unit cell $\Omega^{u}$

$$
\mathcal{R}=\left\{\left(\frac{j_{1} L_{1}}{M_{1}}, \frac{j_{2} L_{2}}{M_{2}}, \frac{j_{3} L_{3}}{M_{3}}\right) \mid j_{i}=0, \ldots, M_{i}-1, \quad i=1,2,3\right\} .
$$

Then each eigenfunction $u_{b, \mathbf{k}}(\mathbf{r})$ is represented as a column vector. With some abuse of notation, this column vector is still denoted by $u_{b, \mathbf{k}} \in \mathbb{C}^{M \times 1}$, and $u_{b, \mathbf{k}}(\mathbf{j}) \equiv u_{b, \mathbf{k}}\left(\mathbf{r}_{\mathbf{j}}\right), \mathbf{r}_{\mathbf{j}} \in \mathcal{R}$.

Since the effectiveness of the technique presented in this paper is heavily based on numerical linear algebra procedures such as QRCP factorizations and discrete Fourier transforms, it turns out that using discrete variable indices such 
as $\mathbf{j}$ is more convenient than the continuous variable indices such as $\mathbf{r}$. Therefore we will use discrete indices when possible for the rest of the paper. Furthermore, we let

$$
\Omega^{u}=\left\{\left(j_{1}, j_{2}, j_{3}\right) \mid j_{i}=0, \ldots, M_{i}-1, \quad i=1,2,3\right\}
$$

denote the set of indices corresponding to real space grid points $\mathcal{R}$ in the unit cell. The periodic boundary condition on $\Omega^{u}$ allows us to interpret $\mathbf{j}$ and $\mathbf{j}+M_{i} \mathbf{e}_{i}$ as equivalent points $(i=1,2,3)$. The periodic eigenvector $u_{b, \mathbf{k}}$ satisfies the discrete orthonormal condition

$$
\sum_{\mathbf{j} \in \Omega^{u}} u_{b, \mathbf{k}}^{*}(\mathbf{j}) u_{b^{\prime}, \mathbf{k}^{\prime}}(\mathbf{j})=\delta_{b, b^{\prime}} \delta_{\mathbf{k}, \mathbf{k}^{\prime}}
$$

Again, we denote by

$$
\Omega^{g}=\left\{\left(j_{1}, j_{2}, j_{3}\right) \mid j_{i}=0, \ldots, N_{i} M_{i}-1, \quad i=1,2,3\right\}
$$

the corresponding set of indices of real space grid points in the global supercell. Similar to before, the periodic boundary condition on $\Omega^{g}$ allows us to interpret $\mathbf{j}$ and $\mathbf{j}+N_{i} M_{i} \mathbf{e}_{i}$ as equivalent points $(i=1,2,3)$. The discretized eigenfunction $\psi_{b, \mathbf{k}}(\mathbf{j})$ is periodic on the global supercell $\Omega^{g}$, and satisfies the discrete orthonormal condition

$$
\sum_{\mathbf{j} \in \Omega^{g}} \psi_{b, \mathbf{k}}^{*}(\mathbf{j}) \psi_{b^{\prime}, \mathbf{k}^{\prime}}(\mathbf{j})=\delta_{b, b^{\prime}} \delta_{\mathbf{k}, \mathbf{k}^{\prime}} N
$$

Note that the convention taken in Eq. (10) places the origin of the unit cell $\Omega^{u}$ at the origin of the global supercell $\Omega^{g}$ as well. This is allowed due to the periodicity of the global supercell.

We now introduce a key concept for the development of our algorithm, the discrete density matrix. Notationally, it is denoted by $P_{\mathbf{k}} \in \mathbb{C}^{(M N) \times(M N)}$ and for each $\mathbf{k}$-point is defined as

$$
P_{\mathbf{k}}\left(\mathbf{j}, \mathbf{j}^{\prime}\right)=\frac{1}{N} \sum_{b=1}^{n_{b}} \psi_{b, \mathbf{k}}(\mathbf{j}) \psi_{b, \mathbf{k}}^{*}\left(\mathbf{j}^{\prime}\right), \quad \mathbf{j}, \mathbf{j}^{\prime} \in \Omega^{g}
$$

Here $*$ stands for the Hermitian conjugate operation. The complete discrete density matrix is then defined as

$$
P\left(\mathbf{j}, \mathbf{j}^{\prime}\right)=\sum_{\mathbf{k} \in \mathcal{K}} P_{\mathbf{k}}\left(\mathbf{j}, \mathbf{j}^{\prime}\right), \quad \mathbf{j}, \mathbf{j}^{\prime} \in \Omega^{g} .
$$

It is easy to verify that $P_{\mathbf{k}}$ and $P$ satisfies the normalization conditions

$$
\operatorname{Tr} P_{\mathbf{k}}=n_{b} \quad \text { and } \quad \operatorname{Tr} P=N n_{b}
$$

The following block circulant property of the density matrix plays an important role in the SCDM-k method for constructing localized orbitals. 
Proposition 1. P satisfies the block circulant property, i.e.

$$
P\left(\mathbf{j}+M_{i} \mathbf{e}_{i}, \mathbf{j}^{\prime}+M_{i} \mathbf{e}_{i}\right)=P\left(\mathbf{j}, \mathbf{j}^{\prime}\right), \quad \forall \mathbf{j}, \mathbf{j}^{\prime} \in \Omega^{g}, \quad i=1,2,3 .
$$

Proof. It is sufficient to show that each $P_{\mathbf{k}}$ satisfies the block circulant property. For any $i=1,2,3$,

$$
\begin{aligned}
& P_{\mathbf{k}}\left(\mathbf{j}+M_{i} \mathbf{e}_{i}, \mathbf{j}^{\prime}+M_{i} \mathbf{e}_{i}\right)=\frac{1}{N} \sum_{b=1}^{n_{b}} \psi_{b, \mathbf{k}}\left(\mathbf{j}+M_{i} \mathbf{e}_{i}\right) \psi_{b, \mathbf{k}}^{*}\left(\mathbf{j}^{\prime}+M_{i} \mathbf{e}_{i}\right) \\
= & \frac{1}{N} \sum_{b=1}^{n_{b}} e^{\imath \mathbf{k} \cdot\left(\mathbf{r}_{\mathbf{j}}+L_{i} \mathbf{e}_{i}\right)} u_{b, \mathbf{k}}\left(\mathbf{j}+M_{i} \mathbf{e}_{i}\right) e^{-\imath \mathbf{k} \cdot\left(\mathbf{r}_{\mathbf{j}^{\prime}}+L_{i} \mathbf{e}_{i}\right)} u_{b, \mathbf{k}}^{*}\left(\mathbf{j}^{\prime}+M_{i} \mathbf{e}_{i}\right) \\
= & e^{\imath \mathbf{k} \cdot \mathbf{r}_{\mathbf{j}}} u_{b, \mathbf{k}}(\mathbf{j}) e^{-\imath \mathbf{k} \cdot \mathbf{r}_{\mathbf{j}^{\prime}}} u_{b, \mathbf{k}}^{*}\left(\mathbf{j}^{\prime}\right)=P_{\mathbf{k}}\left(\mathbf{j}, \mathbf{j}^{\prime}\right) .
\end{aligned}
$$

Therefore, each $P_{\mathbf{k}}$ is block circulant. Here we have implicitly used the aforementioned periodic structure over $\Omega^{g}$ to address when $\mathbf{j}+M_{i} \mathbf{e}_{i}$ or $\mathbf{j}^{\prime}+M_{i} \mathbf{e}_{i}$ yields a point outside the boundary of $\Omega^{g}$.

\section{Selected columns of the density matrix}

Once the Kohn-Sham equations have been solved numerically, we have the means to construct a set of $N n_{b}$ eigenfunctions encoded as columns of $\Psi^{g}$ over the global supercell $\Omega^{g}$. However, the functions will be delocalized spatially. We now outline a construction for computing $N n_{b}$ localized eigenfunctions over the global supercell that span the same space as $\Psi^{g}$. Notably, the matrix of all $N n_{b}$ vectors over $N M$ spatial points may be prohibitively expensive to even store. As a consequence of this, we provide algorithms that construct $n_{b}$ of these localized functions associated with a single unit cell. The periodic structure of the problem means this is sufficient for our needs.

\subsection{Review of the SCDM procedure for $\Gamma$ point calculation}

In order to present the SCDM-k method, we first briefly review the procedure for localizing Kohn-Sham orbitals via the SCDM procedure for $\Gamma$ point calculations, of which the details can be found in Ref. [16]. In order to remain notationally consistent within this work, we use slightly different notation than in $[16]$.

We present the SCDM method as if Kohn-Sham orbitals are only defined on a single unit cell $\Omega^{u}$ with the one $\mathbf{k}$ point, the so-called the $\Gamma$ point, i.e. $N=1$. Let $\left\{\psi_{\alpha}\right\}_{\alpha=1}^{n_{b}}$ represent the $n_{b}$ Kohn-Sham orbitals discretized on a uniform grid, and collected as columns of the matrix $\Psi$. We leverage the fact that the density matrix $P=\Psi \Psi^{*}$ has well localized columns for insulating systems [19], and use columns of $P$ as a starting point for constructing a localized basis. We are always interested in finding a representation of the entire Kohn-Sham invariant subspace and thus construct $n_{b}$ localized orbitals. 
Algorithm 1 presents the SCDM algorithm in its simplest form, providing a unitary transform from $\Psi$ to a set of orthogonal localized orbitals $\Phi$. Here we see that the algorithm computationally amounts to a single QRCP factorization. This factorization can be computed, e.g., via the qr function in MATLAB [29] or the DGEQP3 routine in LAPACK [30].

To motivate the algorithmic developments here, we also present a slight variation on Algorithm 1. Specifically, we assume that we do not have access to the matrix Q. Instead we simply have a set of $n_{b}$ columns that the permutation matrix $\Pi$ chose to move forward during the QRCP process. It turns out that this information is sufficient to generate a localized basis. This variation is presented in Algorithm 2, where we first compute the set $\mathcal{C}$ and then construct the relevant columns of $\Psi \Psi^{*}$. These columns are themselves well localized but they are not orthogonal, which is a desirable property. Fortunately, because

$$
\Psi \Psi=P_{:, \mathcal{C}}\left(P_{\mathcal{C}, \mathcal{C}}\right)^{-1} P_{:, \mathcal{C}}^{*}
$$

we may orthogonalize $P_{:, \mathcal{C}}$ using the square root of $\left(P_{\mathcal{C}, \mathcal{C}}\right)^{-1}$. The rapid decay away from the diagonal of $P_{\mathcal{C}, \mathcal{C}}$ ensures that the resulting orthogonalized columns remain well localized.
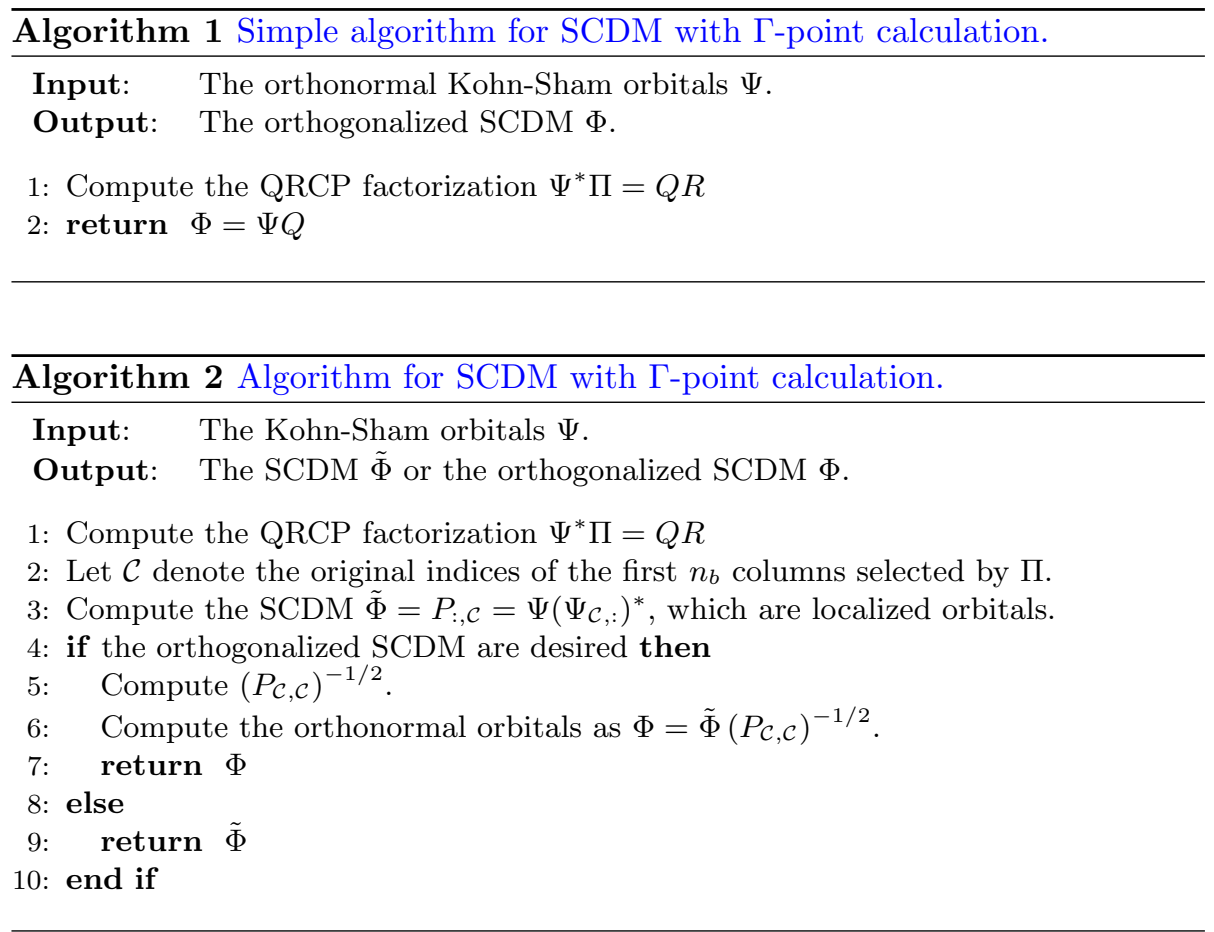

\subsection{SCDM with Brillouin zone sampling}

When we had a single k-point we sought to compute a set of $n_{b}$ localized functions in the cell associated with that $\mathbf{k}$-point. Now, we may view our spatial 
domain as consisting of $N$ unit cells, consequently we seek to compute $N n_{b}$ localized functions over the entire spatial domain $\Omega^{g}$. The most straightforward way to accomplish this would be to simply treat a larger problem, one with $N M$ spatial unknowns and $N n_{b}$ eigenfunctions denoted by $\Psi^{g}$, as input to Algorithm 1 or 2 . In this case, the global density matrix $\Psi^{g}\left(\Psi^{g}\right)^{*}$ would exhibit the locality we desire. However, this could be prohibitively expensive since the QRCP computation would scale as $\mathcal{O}\left(N^{3}\right)$. However, conceptually such a procedure can serve as a point of comparison for the performance of our new algorithm.

To overcome this obstacle, we appeal to the block circulant property in Proposition 1. Applying our algorithm to $\Psi^{g}$ directly we would expect that the index set $\mathcal{C}$, of size $N n_{b}$, would contain $n_{b}$ columns associated with each unit cell $\Omega^{u}$. Therefore, we will solve a smaller problem to extract $n_{b}$ columns corresponding to a single unit cell. We then construct a set denoted $\mathcal{C}^{g}$ that may be used for the entire global supercell by simply adding the translates of these columns into the other unit cells, with $N$ unique translates of the $n_{b}$ columns this yields a set of the desired size. Once we have this set of columns of the global density matrix, we leverage the block circulant structure of $P$ to efficiently perform the orthogonalization in a manner analogous to lines 6 and 7 of Algorithm 3.

The set $\mathcal{C}^{g}$ constructed by the above procedure does not necessarily coincide with the columns of $P$ we would select if we used our existing algorithm directly on the global problem. However, we make the assumption that while the detailed shape of the columns of the density matrix requires a relatively large number of k-points to resolve, the actually selection of columns in a given unit cell is relatively insensitive to the number of $\mathbf{k}$-points used. Since $u_{b, \mathbf{k}}$ is discretized on a fine real space grid, even if the columns selected shift by a few grid points the resulting columns of the global density matrix should still be well localized. Numerical experiments in section 5 corroborate this intuitive argument.

We now explicitly introduce the small local supercell $\Omega^{\ell}$ where $\Omega^{u} \subset \Omega^{\ell} \subset \Omega^{g}$ (Figure 1) used in to pick the $n_{b}$ columns of $\mathcal{C}$ in a single unit cell. With a similar abuse of notation as before, $\Omega^{\ell}$ is also used to denote the indices of grid points in the local supercell. The number of unit cells in $\Omega^{\ell}$ along the $i$-th direction is denoted by $N_{i}^{\ell}$. Following the same convention as in the definition of the global supercell in Eq. (10), the grid points in $\Omega^{\ell}$ are

$$
\Omega^{\ell}=\left\{\left(j_{1}, j_{2}, j_{3}\right) \mid j_{i}=0, \ldots, N_{i}^{\ell} M_{i}-1, \quad i=1,2,3\right\},
$$

which places the origin of the unit cell $\Omega^{\ell}$ also at the origin of the global supercell $\Omega^{g}$.

\subsection{Computing columns of the density matrix}

We now discuss the construction of selected columns of the density matrix that are localized. Let $q_{i}=N_{i} / N_{i}^{\ell}$. If $q_{i}$ is an integer for $i=1,2,3$ then the Kohn-Sham equations on the local supercell $\Omega^{\ell}$ can be simply solved through a 
coarse sampling of the Brillouin zone. The resulting collection of grid points in the Brillouin zone is denoted by

$$
\mathcal{K}^{\ell}=\left\{\left(\frac{2 \pi j_{1}}{N_{1}^{\ell} L_{1}}, \frac{2 \pi j_{2}}{N_{2}^{\ell} L_{2}}, \frac{2 \pi j_{3}}{N_{3}^{\ell} L_{3}}\right) \mid j_{i}=-\frac{N_{i}^{\ell}}{2}+1, \ldots, \frac{N_{i}^{\ell}}{2}, \quad i=1,2,3\right\} .
$$

Since the local supercell is only used as a numerical tool to select columns more efficiently, we make an additional approximation by discarding the shift vector $\mathbf{s}$ when reconstructing the Kohn-Sham orbital $\psi_{b, \mathbf{k}}^{\ell}$ from its periodic part $u_{b, \mathbf{k}}$, and all $\psi_{b, \mathbf{k}}^{\ell}$ 's satisfying the BvK boundary condition in $\Omega^{\ell}$.

After solving the Kohn-Sham equations on the local supercell via coarse sampling of the Brillouin zone, we get $N^{\ell} n_{b}$ orthonormal wavefunctions and arrange them into the columns of the $N^{\ell} M \times N^{\ell} n_{b}$ matrix $\Psi^{\ell}$. We now apply the SCDM procedure for $\Gamma$-point calculations as outlined in Algorithm 2 to $\Psi^{\ell}$, which selects $n_{b} N^{\ell}$ columns denoted $\mathcal{C}^{\ell}$. We then restrict this larger set of selected columns to the set $\mathcal{C}^{u}$ which contains the $n_{b}$ columns associated with points inside a single unit cell $\Omega^{u}$.

Given $\mathcal{C}^{u}$, we may compute the respective selected columns of the density matrix on the entire global supercell as $P(\mathbf{j}, \mathbf{c}), \mathbf{j} \in \Omega^{g}, \mathbf{c} \in \mathcal{C}^{u}$. We first construct $P_{\mathbf{k}}(\mathbf{j}, \mathbf{c}), \mathbf{j} \in \Omega^{u}, \mathbf{c} \in \mathcal{C}^{u}$ as

$$
P_{\mathbf{k}}(\mathbf{j}, \mathbf{c})=\frac{1}{N} \sum_{b=1}^{n_{b}} e^{\imath \mathbf{k} \cdot\left(\mathbf{r}_{j}-\mathbf{r}_{c}\right)} u_{b, \mathbf{k}}(\mathbf{j}) u_{b, \mathbf{k}}^{*}(\mathbf{c}) .
$$

Then for $\mathbf{j} \in \Omega^{g} \backslash \Omega^{u}$, note that for any $n_{i}=0, \ldots, N_{i}-1, i=1,2,3$, we have

$$
\begin{aligned}
P_{\mathbf{k}}\left(\mathbf{j}+n_{i} M_{i} \mathbf{e}_{i}, \mathbf{c}\right) & =e^{\imath \mathbf{k} \cdot\left(n_{i} L_{i} \mathbf{e}_{i}\right)}\left(\frac{1}{N} \sum_{b=1}^{n_{b}} e^{\imath \mathbf{k} \cdot\left(\mathbf{r}_{j}-\mathbf{r}_{c}\right)} u_{b, \mathbf{k}}\left(\mathbf{j}+n_{i} M_{i} \mathbf{e}_{i}\right) u_{b, \mathbf{k}}^{*}(\mathbf{c})\right) \\
& =e^{\imath \mathbf{k} \cdot\left(n_{i} L_{i} \mathbf{e}_{i}\right)} P_{\mathbf{k}}(\mathbf{j}, \mathbf{c}) .
\end{aligned}
$$

Therefore, $P_{\mathbf{k}}$ can be constructed just by multiplying $P_{\mathbf{k}}(\mathbf{j}, \mathbf{c}), \mathbf{j} \in \Omega^{u}$ by phase factors. Summing up $P_{\mathbf{k}}(\mathbf{j}, \mathbf{c})$ 's for all $\mathbf{k}$ we obtain $P(\mathbf{j}, \mathbf{c})$. For convenience we let $P_{\mathcal{C}} \in \mathbb{C}^{(M N) \times n_{b}}$ denote the matrix elements

$$
P_{:, \mathcal{C}^{u}}(\mathbf{j}, b)=P\left(\mathbf{j}, \mathbf{c}_{b}\right), \quad \mathbf{j} \in \Omega^{g}, \mathbf{c}_{b} \in \mathcal{C}^{u} .
$$

The preceding discussion is summarized in Algorithm 3 and yields the desired selected columns of the density matrix over the global supercell. Note that this corresponds to computing only $n_{b}$ of the $N n_{b}$ total localized functions we expect. If desired, the others may be constructed by translating $\mathcal{C}^{u}$ into a different unit cell, see the following section for details.

\subsection{Construct the orthonormalized SCDM}

We now have a procedure to compute $\mathcal{C}^{u}$ and due to the block circulant property of the density matrix, this is sufficient. All the remaining columns are 
$\overline{\text { Algorithm } 3 \text { Computing the (non-orthogonal) selected columns of the density }}$ matrix inside a unit cell.

Input: $\quad$ Monkhorst-Pack points in the Brillouin zone $\mathcal{K}$;

Periodic parts of Kohn-Sham orbitals $\left\{u_{n \mathbf{k}}\right\}$ for $n=1, \ldots, n_{b}$ and $\mathbf{k} \in \mathcal{K}$;

Sub-sampling Monkhorst-Pack points in the reciprocal space $\mathcal{K}^{\ell}$;

Output: Non-orthogonal SCDM associated with the unit cell $\Omega^{u}$.

1: Construct $\Psi^{\ell}$ from $\left\{u_{n \mathbf{k}}\right\}$ with $\mathbf{k} \in \mathcal{K}^{\ell}$ from the Bloch decomposition (4).

2: Compute the QRCP factorization $\left(\Psi^{\ell}\right)^{*} \Pi=Q R$

3: Let $\mathcal{C}^{\ell}$ denote the original indices of the first $N^{\ell} n_{b}$ columns selected by $\Pi$.

4: Find the selected column indices $\mathcal{C}^{u}=\left\{j \in \mathcal{C}^{\ell} \mid j \in \Omega^{u}\right\}$ in the unit cell.

5: for all $\mathrm{k}$ do

6: Construct $P_{\mathbf{k}}(\mathbf{j}, \mathbf{c})$ for $\mathbf{j} \in \Omega^{u}$ and $\mathbf{c} \in \mathcal{C}^{u}$ via (16).

7: end for

8: Compute $P(\mathbf{j}, \mathbf{c})$ for $\mathbf{j} \in \Omega^{g}$ and $\mathbf{c} \in \mathcal{C}^{u}$ using (17).

the block translates of these columns into the other unit cells in $\Omega^{g}$. Define the collection of indices

$$
\mathcal{C}^{g} \equiv\left\{\mathbf{c}+\left(n_{1} M_{1}, n_{2} M_{2}, n_{3} M_{3}\right) \mid \mathbf{c} \in \mathcal{C}, \quad n_{i}=0, \ldots, N_{i}-1, \quad i=1,2,3\right\},
$$

which induces a matrix $P_{:, \mathcal{C}^{g}} \in \mathbb{C}^{(M N) \times\left(n_{b} N\right)}$ such that

$$
P_{:, \mathcal{C}^{g}}(\mathbf{j}, b)=P\left(\mathbf{j}, \mathbf{c}_{b}\right), \quad \mathbf{j} \in \Omega^{g}, \mathbf{c}_{b} \in \mathcal{C}^{g} .
$$

These columns of $P$ are precisely the $N n_{b}$ columns that form our localized basis over the entire global supercell. However, they are not orthonormal and we now describe an efficient procedure to orthonormalize them.

The matrix $P_{:, \mathcal{C}^{g}}$ is block circulant when viewed as an $N \times N$ block matrix with each block of size $M \times n_{b}$. Note that the storage cost of $P_{\mathcal{C}^{g}}$ is $\mathcal{O}\left(n_{b} M N^{2}\right)$, and it is therefore never explicitly computed or stored. We also define the matrix $P_{\mathcal{C}^{g}, \mathcal{C}^{g}} \in \mathbb{C}^{\left(n_{b} N\right) \times\left(n_{b} N\right)}$ as

$$
P_{\mathcal{C}^{g}, \mathcal{C}^{g}}(a, b)=P\left(\mathbf{c}_{a}, \mathbf{c}_{b}\right), \quad \mathbf{c}_{a}, \mathbf{c}_{b} \in \mathcal{C}^{g}
$$

and note that $P_{\mathcal{C}^{g}, \mathcal{C}^{g}}$ is also block circulant when viewed as an $N \times N$ block matrix with each block of size $n_{b} \times n_{b}$.

Now, if we consider the matrix $\Phi^{g} \in \mathbb{C}^{(M N) \times\left(n_{b} N\right)}$ defined as

$$
\Phi^{g}=P_{:, \mathcal{C}^{g}}\left(P_{\mathcal{C}^{g}, \mathcal{C}^{g}}\right)^{-\frac{1}{2}},
$$

it is also block circulant and satisfies the discrete orthonormality condition

$$
\left(\Phi^{g}\right)^{*} \Phi^{g}=I
$$

Therefore, $\Phi^{g}$ represents an orthonormal set of localized basis functions across all of the k-points. Due to the translational invariance, we only need to compute 
the columns of $\Phi^{g}$ centered in $\Omega^{u}$, and as before, the remaining columns are just the block translates of these columns into the other unit cells in $\Omega^{g}$. Similar to $P_{:, \mathcal{C}^{g}}$, the entire matrix $\Phi^{g}$ is neither explicitly constructed nor stored in practice.

In fact, all of the rows of $P_{:, \mathcal{C}^{g}}$ and $\Phi^{g}$ may be constructed from knowledge of the first $M$ rows, i.e. those associated with a single unit cell. More specifically, if $\mathbf{j} \in \Omega^{u}$ and $\mathbf{j}^{\prime} \in \Omega^{g}$ are such that

$$
\mathbf{j}^{\prime}=\mathbf{j}+\left(n_{1} M_{1}, n_{2} M_{2}, n_{3} M_{3}\right)
$$

for some $\left(n_{1}, n_{2}, n_{3}\right)$, then for any $\mathbf{c}_{b} \in \mathcal{C}^{g}$

$$
\Phi^{g}\left(\mathbf{j}^{\prime}, \mathbf{c}_{b}\right)=\Phi^{g}\left(\mathbf{j}, \mathbf{c}_{b}-\left(n_{1} M_{1}, n_{2} M_{2}, n_{3} M_{3}\right)\right) .
$$

To make this explicit notationally, let $P_{\Omega^{u}, \mathcal{C}^{g}} \in \mathbb{C}^{(M) \times\left(n_{b} N\right)}$ be such that

$$
P_{\Omega^{u}, \mathcal{C}^{g}}(\mathbf{j}, b)=P\left(\mathbf{j}, \mathbf{c}_{b}\right), \quad \mathbf{j} \in \Omega^{u}, \mathbf{c}_{b} \in \mathcal{C}^{g} .
$$

We define $\Phi^{u}$ as

$$
\Phi^{u}=P_{\Omega^{u}, \mathcal{C}^{g}}\left(P_{\mathcal{C}^{g}, \mathcal{C}^{g}}\right)^{-\frac{1}{2}},
$$

and since we may construct $\Phi^{g}$ from $\Phi^{u}$ via Eq. (19) we now focus on the construction of $\Phi^{u}$.

Direct computation of the matrix square root of $P_{\mathcal{C}^{g}, \mathcal{C}^{g}} \in \mathbb{C}^{\left(n_{b} N\right) \times\left(n_{b} N\right)}$ in Eq. (18) costs $\mathcal{O}\left(N^{3}\right)$ and is hence computationally expensive. Instead, we demonstrate an algorithm to take advantage of the block circulant property of $P_{\mathcal{C}^{g}, \mathcal{C}^{g}}$ that reduces the complexity to $\mathcal{O}(N \log N)$. Let $F$ be the matrix representing the three-dimensional discrete Fourier transform that acts with respect to the $\mathbf{n}=\left(n_{1}, n_{2}, n_{3}\right)$ index in the set $\mathcal{C}^{g}$ and $F^{-1}=F^{*}$ be the threedimensional inverse discrete Fourier transform that acts with respect to the Fourier space grid corresponding to n. Computationally these operations are performed via the fast Fourier transform. Observe that,

$$
\begin{aligned}
\Phi^{u} & =P_{\Omega^{u}, \mathcal{C}^{g}} F^{-1} F\left(P_{\mathcal{C}^{g}, \mathcal{C}^{g}}\right)^{-\frac{1}{2}} F^{-1} F \\
& =\left(P_{\Omega^{u}, \mathcal{C}^{g}} F^{-1}\right)\left(F P_{\mathcal{C}^{g}, \mathcal{C}^{g}} F^{-1}\right)^{-\frac{1}{2}} F .
\end{aligned}
$$

We can move the square root outside $F$ and $F^{-1}$ because $F\left(P_{\mathcal{C}^{g}, \mathcal{C}^{g}}\right)^{-\frac{1}{2}} F^{-1}$ and $\left(F P_{\mathcal{C}^{g}, \mathcal{C}^{g}} F^{-1}\right)^{-\frac{1}{2}}$ have exactly the same eigenvalues and eigenvectors. This is a consequence of the fact that $F$ is unitary and $P_{\mathcal{C}^{g}, \mathcal{C}^{g}}$ is Hermitian positive definite.

Eq. (20) compactly represents the procedure for accelerating the computation of the orthogonalized SCDM and we now elaborate on their construction. First, we observe that the matrix

$$
\hat{P}_{\mathcal{C}^{g}, \mathcal{C}^{g}}=F P_{\mathcal{C}^{g}, \mathcal{C}^{g}} F^{-1}
$$

is block diagonal with $N$ blocks each of size $n_{b} \times n_{b}$. This means that, $\left(\hat{P}_{\mathcal{C}^{g}, \mathcal{C}^{g}}\right)^{-1 / 2}$ may be computed by taking the inverse square root of each small block independently. The block diagonal structure follows immediately from the fact that 
$P_{\mathcal{C}^{g}, \mathcal{C}^{g}}$ is block circulant and the use of the discrete Fourier transform. Furthermore, the diagonal blocks may be computed by applying $F$ to the first $n_{b}$ columns of $P_{\mathcal{C}^{g}, \mathcal{C}^{g}}$, which means those are the only columns we need to explicitly construct. Finally, since there are $N$ diagonal blocks in total, we may associate each of the diagonal blocks with an index $\mathbf{n}$. Therefore, we refer to a given diagonal block of $\hat{P}_{\mathcal{C}^{g}, \mathcal{C}^{g}}$ as $\hat{P}_{\mathcal{C}^{g}, \mathcal{C}^{g} ; \mathbf{n}}$.

We now let

$$
\hat{P}_{\Omega^{u}, \mathcal{C}^{g}}=P_{\Omega^{u}, \mathcal{C}^{g}} F^{-1},
$$

which may be constructed by applying $F$ to the columns of $\left(P_{\Omega^{u}, \mathcal{C}^{g}}\right)^{*}$. Finally, similar to before we let $\hat{P}_{\Omega^{u}, \mathcal{C}^{g} ; \mathbf{n}}$ denote a $M \times n_{b}$ matrix containing $n_{b}$ of the columns of $\hat{P}_{\Omega^{u}, \mathcal{C}^{g}}$ associated with a given index $\mathbf{n}$. We define

$$
\hat{\Phi}^{u}=\Phi^{u} F^{-1},
$$

and once again let $\hat{\Phi}_{\mathbf{n}}^{u}$ denote a $M \times n_{b}$ matrix containing $n_{b}$ columns of $\hat{\Phi}^{u}$ associated with a given index $\mathbf{n}$.

The use of a single index allows us to compactly write the construction of $\hat{\Phi}^{u}$ as

$$
\hat{\Phi}_{\mathbf{n}}^{u}=\hat{P}_{\Omega^{u}, \mathcal{C}^{g} ; \mathbf{n}}\left(\hat{P}_{\mathcal{C}^{g}, \mathcal{C}^{g} ; \mathbf{n}}\right)^{-1 / 2} .
$$

We may then form the first $M$ rows associated with a single unit cell of $\Phi^{g}$ by applying $F^{-1}$ to the columns of $\hat{\Phi}_{\mathbf{n}}^{u}$. Algorithm 4 summarizes the steps for constructing the orthogonalized SCDM centered in a single unit cell.

$\overline{\text { Algorithm } 4 \text { Computing the orthonormalized SCDM from the non-orthogonal }}$ SCDM.

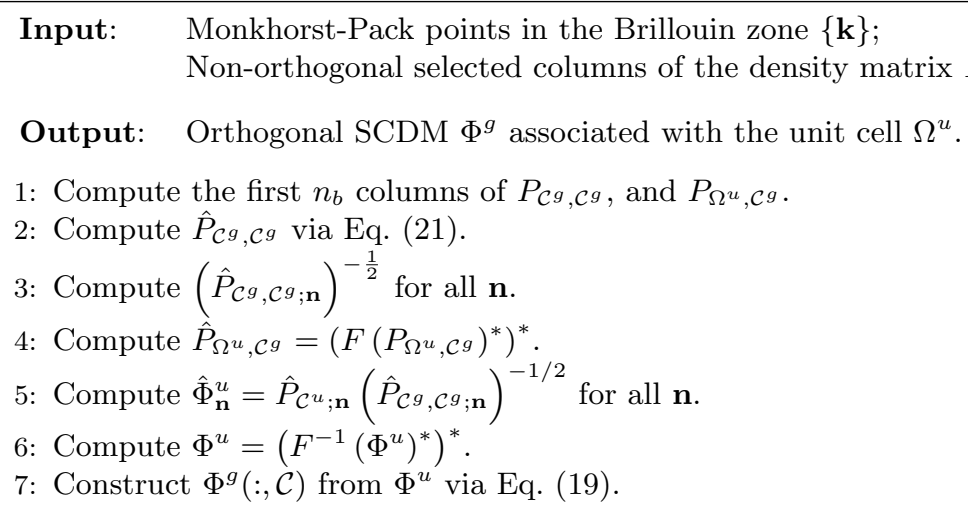

Input: Monkhorst-Pack points in the Brillouin zone $\{\mathbf{k}\}$;

Output: Orthogonal $\operatorname{SCDM} \Phi^{g}$ associated with the unit cell $\Omega^{u}$.

1: Compute the first $n_{b}$ columns of $P_{\mathcal{C}^{g}, \mathcal{C}^{g}}$, and $P_{\Omega^{u}, \mathcal{C}^{g}}$.

2: Compute $\hat{P}_{\mathcal{C}^{g}, \mathcal{C}^{g}}$ via Eq. (21).

3: Compute $\left(\hat{P}_{\mathcal{C}^{g}, \mathcal{C}^{g} ; \mathbf{n}}\right)^{-\frac{1}{2}}$ for all $\mathbf{n}$.

4: Compute $\hat{P}_{\Omega^{u}, \mathcal{C}} g=\left(F\left(P_{\Omega^{u}, \mathcal{C}}\right)^{*}\right)^{*}$

5: Compute $\hat{\Phi}_{\mathbf{n}}^{u}=\hat{P}_{\mathcal{C}^{u} ; \mathbf{n}}\left(\hat{P}_{\mathcal{C}^{g}, \mathcal{C}^{g} ; \mathbf{n}}\right)^{-1 / 2}$ for all $\mathbf{n}$.

6: Compute $\Phi^{u}=\left(F^{-1}\left(\Phi^{u}\right)^{*}\right)^{*}$.

7: Construct $\Phi^{g}(:, \mathcal{C})$ from $\Phi^{u}$ via Eq. (19).

Non-orthogonal selected columns of the density matrix $P_{\mathcal{C}}$;

\subsection{Post-processing for the shift vector in the Monkhorst-Pack grid}

Here we address the issues that arise when using the Monkhorst-Pack grid with half grid shift $\mathbf{s}$. In such case, each orbital $\psi_{\alpha}$ does not satisfy the BvK boundary condition in the global supercell $\Omega^{g}$. Instead,

$$
\psi_{\alpha}\left(\mathbf{r}+N_{i} L_{i} \mathbf{e}_{i}\right)=-\psi_{\alpha}(\mathbf{r}), \quad \forall \mathbf{r} \in \Omega^{g}, \quad i=1,2,3,
$$


and a phase factor $(-1)$ is gained. To be accurate, this phase factor needs to be taken into account in the SCDM. Note that

$$
\widetilde{P}(\mathbf{j}, \mathbf{c})=\sum_{\mathbf{k}} e^{\imath \mathbf{s} \cdot\left(\mathbf{r}_{\mathbf{j}}-\mathbf{r}_{\mathbf{c}}\right)} P_{\mathbf{k}}(\mathbf{j}, \mathbf{c})=e^{\imath \mathbf{s} \cdot\left(\mathbf{r}_{\mathbf{j}}-\mathbf{r}_{\mathbf{c}}\right)} P(\mathbf{j}, \mathbf{c}) .
$$

Here $P_{\mathbf{k}}$ and $P$ are the density matrices obtained by taking $\mathbf{s}=(0,0,0)$. Therefore the post-processing only requires multiplying each column of the SCDM $P(\mathbf{j}, \mathbf{c})$ and the orthonormalized SCDM $\Phi_{\mathbf{j}, \mathbf{c}}^{g}$ by a phase vector $e^{\imath \mathbf{s} \cdot\left(\mathbf{r}_{\mathbf{j}}-\mathbf{r}_{\mathbf{c}}\right)}$. It is straightforward to verify that the post-processing procedure (26) maintains the orthonormality of the orthonormalized SCDM.

\subsection{Complexity}

The computational complexity for selecting the SCDM using the QRCP factorization with a local supercell is $\mathcal{O}\left(\left(N^{\ell}\right)^{3} M n_{b}^{2}\right)$. Then, the complexity for computing the non-orthonormal SCDM $P_{\Omega^{g}, \mathcal{C}^{u}}$ via matrix-matrix multiplication is $\mathcal{O}\left(M N n_{b}^{2}\right)$. Due to the usage of Eq. (17) the cost for computing $P_{\mathcal{C}}(\mathbf{j}, \mathbf{c}), \mathbf{j} \in \Omega^{g}$ is only $\mathcal{O}\left(M N n_{b}\right)$. Importantly, $M$ and $n_{b}$ are assumed to be fixed with respect to the increase of the number of $\mathbf{k}$-points (i.e. the number of unit cells contained in the global supercell). Furthermore, we explicitly set $N^{\ell}$ to be small and not grow with $N$. Therefore, the cost for obtaining the non-orthogonal SCDM is $\mathcal{O}(N)$.

In order to generate the orthonormal SCDM, the cost for computing the Fourier transform $F P_{\mathcal{C}^{g}, \mathcal{C}^{g}} F^{-1}$ is $\mathcal{O}\left(N \log (N) n_{b}^{2}\right)$, and the cost for computing the matrix square root of the block diagonal matrix $F P_{\mathcal{C}^{g}, \mathcal{C}^{g}} F^{-1}$ is $\mathcal{O}\left(N n_{b}^{3}\right)$. The cost for computing the first block row of $\left(P_{\mathcal{C}^{g}} F^{-1}\right)$ is $\mathcal{O}\left(N \log (N) M n_{b}\right)$ and the cost for multiplying with matrix square root is $\mathcal{O}\left(N M n_{b}^{2}\right)$. Therefore, the total cost for generating $\Phi^{g}(\mathbf{j}, \mathbf{c}), \mathbf{j} \in \Omega^{g}, \mathbf{c} \in \Omega^{u}$ is

$$
\left.\mathcal{O}\left(N \log (N) n_{b}^{2}+N n_{b}^{3}+N \log (N) M n_{b}+N M n_{b}^{2}\right)\right) .
$$

Finally, the cost for the post-processing by multiplying a phase vector is $\mathcal{O}\left(M N n_{b}\right)$. If we take the leading term with respect to $N, M$ and think of $n_{b}$ as a small constant, then the complexity of the whole algorithm is

$$
\mathcal{O}\left(N \log (N) M n_{b}+N M n_{b}^{2}+\left(N^{\ell}\right)^{3} M n_{b}^{2}\right) .
$$

Hence, the complexity for obtaining the orthonormalized SCDM with respect to the number of k-points used for sampling the Brillouin zone is only $\mathcal{O}(N \log N)$.

\section{Numerical examples}

To illustrate the performance of the SCDM-k algorithm, we consider the localization of orbitals obtained from model potentials in two and three dimensions. In three dimensions, the model potential takes the form

$$
V(\mathbf{r})=\sum_{n_{1}=0}^{N_{1}-1} \sum_{n_{2}=0}^{N_{2}-1} \sum_{n_{3}=0}^{N_{3}-1} V_{u}\left(\mathbf{r}-\sum_{i=1}^{3} n_{i} \mathbf{e}_{i}\right) .
$$


Here $V_{u}(\mathbf{r})$ is taken to be a Gaussian potential centered at the origin in the unit cell $\Omega^{u}$ modeling an atom, i.e.

$$
V_{u}(\mathbf{r})=-4.0 e^{-\frac{\|\mathbf{r}\|^{2}}{2 \sigma^{2}}} .
$$

The shape of the model potential in two dimensions is similar,

$$
V(\mathbf{r})=\sum_{n_{1}=0}^{N_{1}-1} \sum_{n_{2}=0}^{N_{2}-1} V_{u}\left(\mathbf{r}-\sum_{i=1}^{2} n_{i} \mathbf{e}_{i}\right) .
$$

We generally set $\sigma=1$ and will explicitly state when we use a different value.

\subsection{Shapes of the SCDM}

We first present numerical examples illustrating the shapes of the SCDM in two and three dimensions. In the two dimensional case, we set $L_{1}=L_{2}=6.0$, $M_{1}=M_{2}=32, n_{b}=3$, and use $N_{1}=N_{2}=8 \mathbf{k}$-points per direction. For this example we set $\sigma=0.8$. Setting $n_{b}=3$ means that we should have one orbital that behaves similar to an $s$-orbital (spherical) and two orbitals that behave similar to a $p$-orbital (non-spherical). Figure 2 shows the shape of SCDM and the orthonormalized SCDM. We only plot the three SCDM for a single k-point near the middle of the domain and observe the rapid decay of both the SCDM and the orthogonalized SCDM away from their unit cell.

In three dimensions, we use $N_{1}=N_{2}=N_{3}=4$ k-points per direction, set $M_{1}=M_{2}=M_{3}=20$, and let $n_{b}=4$. Similar to the 2D case, we expect that there should be one orbital that behaves similar to an $s$-orbital and three orbitals that behave similar to a $p$-orbital. Figure 2 shows the SCDM and the orthogonalized SCDM. Here we only plot isosurfaces for the four SCDM for a single $\mathbf{k}$-point near the middle of the domain. As in the two dimensional case, we see that the bulk of the orbital is well localized within a single unit cell. It is also even more apparent than in the $2 \mathrm{~d}$ case that our localized functions match the expected $s$ - and $p$-orbital structure.

In both of the preceding cases, the well localized orbitals also imply that the matrix $P_{\mathcal{C}^{g}, \mathcal{C}^{g}}$ and hence the matrix $F P_{\mathcal{C}^{g}, \mathcal{C}^{g}} F^{-1}$ are well conditioned, and the computation of the matrix square root does not cause any numerical problems. In fact, the condition number of the small block matrices we have to take the inverse square root of was less than five in the two dimensional example and 15 for the three dimensional example.

\subsection{Locality of the $S C D M$}

Figure 2 and 3 show that the SCDM are qualitatively very well localized. We now quantify this by measuring the locality of the functions systematically. Specifically, we construct the SCDM and the orthogonalized SCDM and then, over the global supercell $\Omega^{g}$, measure the fraction of entries (denoted by $n z \%$ ) where the relative magnitude of the functions is above a given threshold $\epsilon$.

First, we measure the locality for the two dimensional problem. We use $N_{1}=N_{2}=16$ k-points in each direction, $n_{b}=3$, and $M_{1}=M_{2}=40$. 

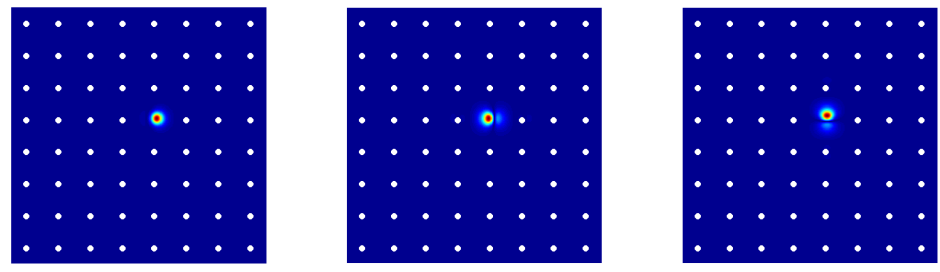

(a)
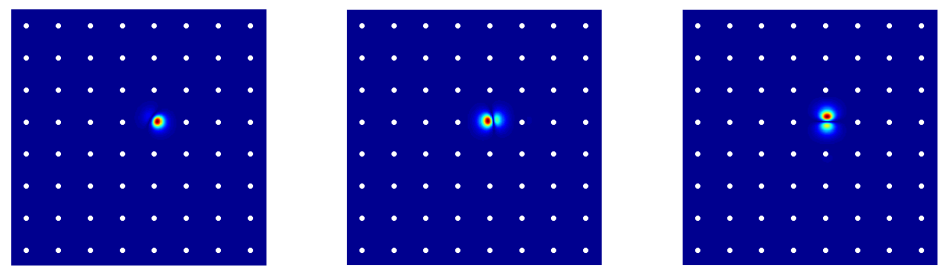

(b)
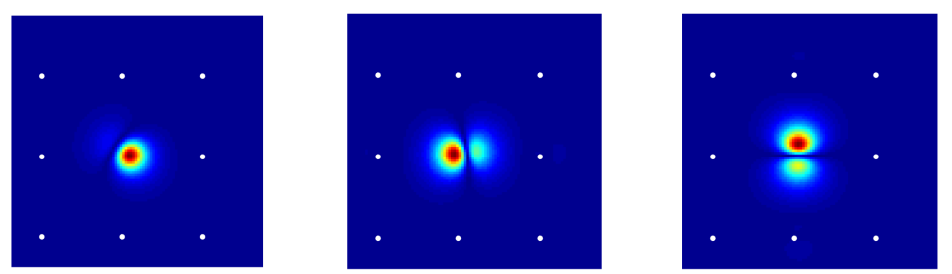

(c)

Figure 2: Absolute value of the three SCDM (a) and orthogonalized SCDM (b) located in a single unit cell and plotted over the global supercell. Zoomed-in images of the significant regions of orthogonalized SCDM (c) showing the expected spherical (s-orbital like) and nonspherical ( $p$-orbitals like) structure.

Figure 4 shows the average locality for both the SCDM and the orthogonalized SCDM. The diameter of the localized region is proportional to the square root of the volume, and thus is proportional to $(n z \%)^{1 / 2}$, consequently this is the quantity we choose to plot. Importantly, here we see that the orthogonalization does not severely impact the localization properties of the SCDM. Furthermore, when the relative truncation threshold is set to $\epsilon=10^{-2}$ for the orthogonalized 


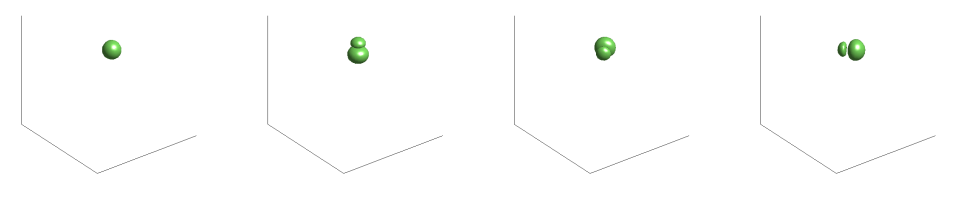

(a)

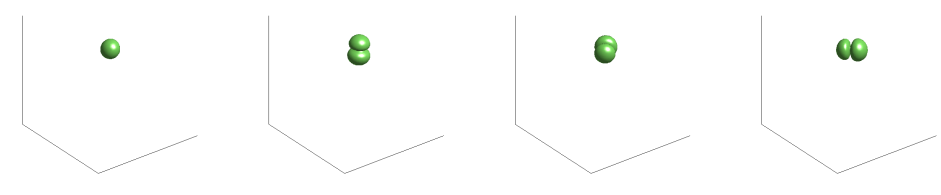

(b)

Figure 3: Isosurface at a relative value of 0.1 of the absolute value of the SCDM (a) and orthogonalized SCDM (b) located in a single unit cell and plotted over the global supercell.

SCDM less than $1 \%$ of the entries remain non-zero. While in some cases here the orthogonalized orbitals are actually more localized, we do not necessarily expect such behavior in general and only expect that the orthogonalization step will not significantly reduce the locality.

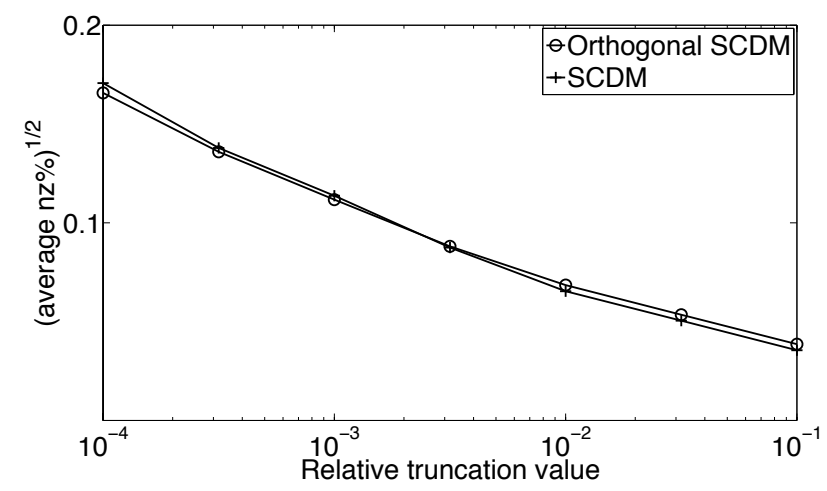

Figure 4: Average fraction of nonzero entries after truncation for the SCDM and the orthogonalized SCDM in two dimensions.

We now move back to the three dimensional problem and use $N_{1}=N_{2}=$ $N_{3}=8$ k-points in each direction, $n_{b}=4$, and $M_{1}=M_{2}=M_{3}=20$. In Figure 5 we plot average locality for both the SCDM and the orthogonalized SCDM. Analogously to before, the diameter of the localized region is proportional to the cube root of the volume, and thus we choose to plot $(n z \%)^{1 / 3}$. Once again, the orthogonalization does not severely impact the localization properties of the SCDM and when the relative truncation threshold is set to $10^{-2}$ only about 
$0.7 \%$ of the entries or the orthogonalized SCDM remain non-zero.

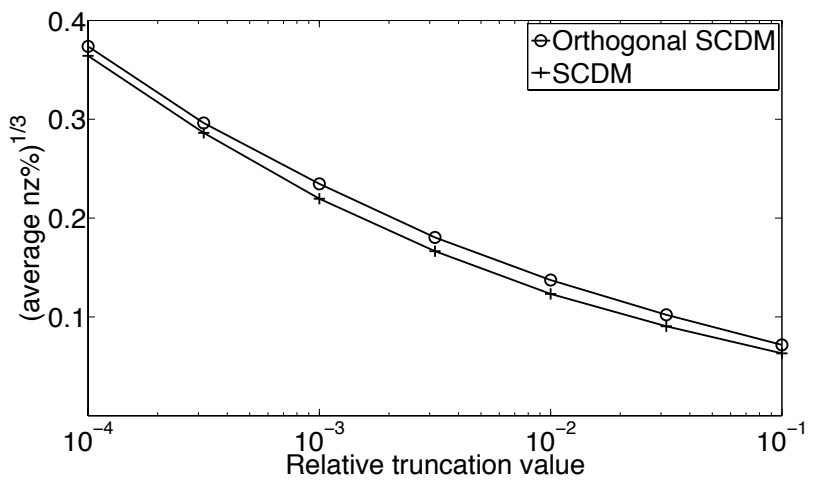

Figure 5: Average fraction of nonzero entries after truncation for the SCDM and the orthogonalized SCDM in three dimensions.

\subsection{Changing the local super cell size}

Rather than finding the selected columns using wavefunctions defined on the entire global supercell $\Omega^{g}$, we find a good approximation to these columns by using a much smaller local supercell $\Omega^{\ell}$, whose size does not increase as the size of the global supercell grows. In fact, this is the key approximation made by our algorithm, and the only source of "error" when compared to our existing methods. Here we quantitatively study the dependence of the quality of the columns selected by this local supercell approach.

Concretely, we use a two dimensional problem with $N_{1}=N_{2}=16$ k-points in each direction, $n_{b}=3$, and $M_{1}=M_{2}=20$. We proceeded to vary the size of the local supercell, $N_{1}^{\ell}$ and $N_{2}^{\ell}$, and compute the SCDM and orthogonalized SCDM. We measure the locality as the fraction of non-zero entries after truncation at a relative magnitude of $10^{-2}$. Figure 6 shows that the localization of both the SCDM and the orthogonalized SCDM is nearly constant as the local supercell size varies.

To compare against our existing methods and validate our approximation, we let the local supercell size grow to match that of the global supercell. This corresponds to running Algorithm 2 on the global problem and in Figure 6 occurs at the rightmost point of the plot since $N_{1}=N_{2}=16$ and $N_{1}^{\ell}=N_{2}^{\ell}=16$. Therefore, we observe that there is no noticable error introduced by our new algorithm: we get functions that are just as localized as if we treated the global problem directly.

\subsection{Scaling with the number of $\mathbf{k}$-points}

Finally, we demonstrate the computational scaling outlined in section 4. Here we consider a three dimensional problem and increase the total number of k-points in each direction. In this experiment we used $M_{1}=M_{2}=M_{3}=10$ and 


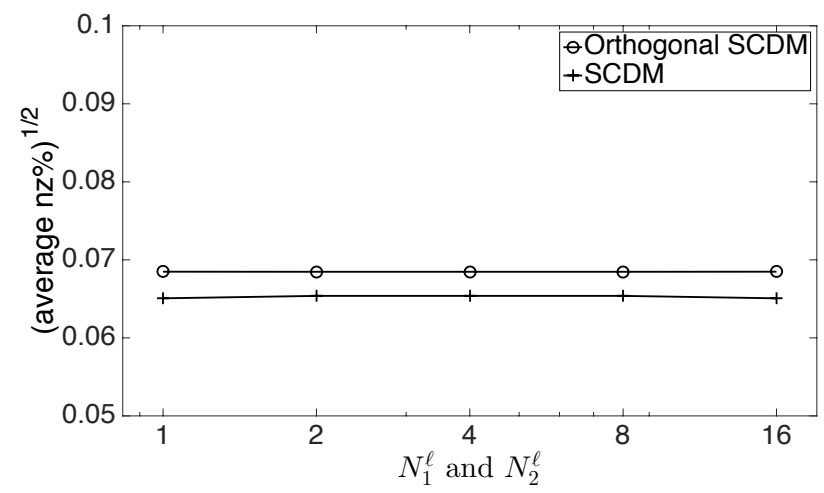

Figure 6: locality as local supercell size grows

$n_{b}=4$. Figure 7 shows the time taken to compute the orthogonalized SCDM as the number of k-points grows. In this case, the terms linear in $N$ actually dominate the computation and we observe close to linear scaling.

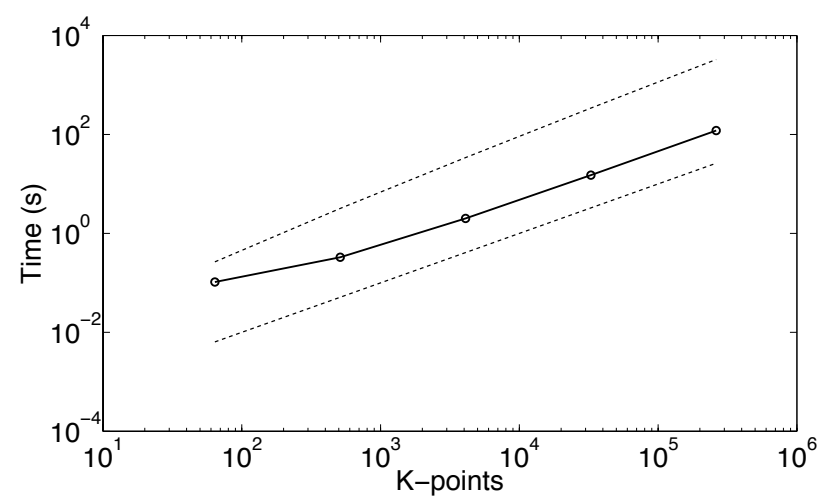

Figure 7: Time taken to compute the orthogonalized SCDM as the number of k-points grows. The upper dotted line represents $\mathcal{O}(N \log N)$ scaling and the lower dotted line represents $\mathcal{O}(N)$ scaling

\section{Conclusion and future work}

We developed the SCDM-k method, which is a new method for finding both orthogonal and non-orthogonal localized orbitals from a set of Kohn-Sham orbitals obtained from Brillouin zone sampling. The SCDM-k method is implicitly based on the use of the gauge invariant density matrix, and obtains localized orbitals without an iterative optimization procedure. Furthermore, the computation exhibits $\mathcal{O}(N \log N)$ scaling with respect to the total number of $\mathbf{k}$-points 
used. Numerical results for two and three dimensional systems with model potentials indicate that the SCDM-k method generates localized orbitals that can be visually similar to MLWFs. All routines used in the SCDM-k method are standard linear algebra routines and are thus easily parallelizable. This could enable efficient computation of localized orbitals for solids both in the postprocessing step and performed on the fly. Though described using a uniform real space grid for example, the SCDM-k method does not rely on a particular basis set and can be combined with any electronic structure software packages supporting k-point sampling in the Brillouin zone. We plan to apply the SCDM$\mathrm{k}$ method to compute localized orbitals and compare directly with MLWFs for Kohn-Sham DFT calculations of real materials systems in the near future.

\section{Acknowledgments}

The work of A.D. is partially supported by a Simons Graduate Research Assistantship. The work of L.L. is partially supported by the DOE Scientific Discovery through the Advanced Computing (SciDAC) program, the DOE Center for Applied Mathematics for Energy Research Applications (CAMERA) program, and by an Alfred P. Sloan fellowship. The work of L.Y. is partially supported by the National Science Foundation under grant DMS-0846501 and the DOE's Advanced Scientific Computing Research program under grant DEFC02-13ER26134/DESC0009409. The authors thank Eric Bylaska, Sinisa Coh, Felipe da Jornada and Bert de Jong for useful discussions, and the anonymous referees for their help in improving this manuscript.

[1] P. Hohenberg, W. Kohn, Inhomogeneous electron gas, Phys. Rev. 136 (1964) B864-B871.

[2] W. Kohn, L. Sham, Self-consistent equations including exchange and correlation effects, Phys. Rev. 140 (1965) A1133-A1138.

[3] J. M. Foster, S. F. Boys, Canonical configurational interaction procedure, Rev. Mod. Phys. 32 (1960) 300-302.

[4] N. Marzari, D. Vanderbilt, Maximally localized generalized Wannier functions for composite energy bands, Phys. Rev. B 56 (20) (1997) 12847-12865.

[5] N. Marzari, A. A. Mostofi, J. R. Yates, I. Souza, D. Vanderbilt, Maximally localized Wannier functions: Theory and applications, Rev. Mod. Phys. 84 (2012) 1419-1475.

[6] X. Wu, A. Selloni, R. Car, Order-N implementation of exact exchange in extended insulating systems, Phys. Rev. B 79 (8) (2009) 085102.

[7] F. Gygi, I. Duchemin, Efficient computation of Hartree-Fock exchange using recursive subspace bisection, J. Chem. Theory Comput. 9 (1) (2012) $582-587$. 
[8] R. D. King-Smith, D. Vanderbilt, Theory of polarization of crystalline solids, Phys. Rev. B 47 (1993) 1651-1654.

[9] S. Goedecker, Linear scaling electronic structure methods, Rev. Mod. Phys. 71 (1999) 1085-1123.

[10] P. Umari, G. Stenuit, S. Baroni, Optimal representation of the polarization propagator for large-scale GW calculations, Phys. Rev. B 79 (20) (2009) 201104.

[11] P. Umari, G. Stenuit, S. Baroni, GW quasiparticle spectra from occupied states only, Phys. Rev. B 81 (2010) 115104.

[12] F. Gygi, Compact representations of Kohn-Sham invariant subspaces, Phys. Rev. Lett. 102 (2009) 166406.

[13] W. E, T. Li, J. Lu, Localized bases of eigensubspaces and operator compression, Proc. Natl. Acad. Sci. 107 (4) (2010) 1273-1278.

[14] V. Ozoliņ̌s, R. Lai, R. Caflisch, S. Osher, Compressed modes for variational problems in mathematics and physics, Proc. Natl. Acad. Sci. 110 (46) (2013) 18368-18373.

[15] F. Aquilante, T. B. Pedersen, A. S. de Merás, H. Koch, Fast noniterative orbital localization for large molecules, J. Chem. Phys. 125 (17) (2006) 174101.

[16] A. Damle, L. Lin, L. Ying, Compressed representation of Kohn-Sham orbitals via selected columns of the density matrix, J. Chem. Theory Comput. 11 (4) (2015) 1463-1469.

[17] W. Kohn, Density functional and density matrix method scaling linearly with the number of atoms, Phys. Rev. Lett. 76 (1996) 3168-3171.

[18] E. Prodan, W. Kohn, Nearsightedness of electronic matter, Proc. Natl. Acad. Sci. 102 (2005) 11635-11638.

[19] M. Benzi, P. Boito, N. Razouk, Decay properties of spectral projectors with applications to electronic structure, SIAM Rev. 55 (1) (2013) 3-64.

[20] E. Blount, Formalisms of band theory, Vol. 13 of Solid State Phys., Academic Press, 1962, pp. 305-373.

[21] J. D. Cloizeaux, Energy bands and projection operators in a crystal: Analytic and asymptotic properties, Phys. Rev. 135 (1964) A685-A697.

[22] J. D. Cloizeaux, Analytical properties of $n$-dimensional energy bands and Wannier functions, Phys. Rev. 135 (1964) A698-A707.

[23] G. Nenciu, Existence of the exponentially localised Wannier functions, Comm. Math. Phys. 91 (1) (1983) 81-85. 
[24] R. Martin, Electronic Structure - Basic Theory and Practical Methods, Cambridge Univ. Pr., West Nyack, NY, 2004.

[25] H. J. Monkhorst, J. D. Pack, Special points for Brillouin-zone integrations, Phys. Rev. B 13 (1976) 5188-5192.

[26] P. Businger, G. H. Golub, Linear least squares solutions by householder transformations, Numerische Mathematik 7 (3) (1965) 269-276.

[27] S. Chandrasekaran, I. C. Ipsen, On rank-revealing factorisations, SIAM Journal on Matrix Analysis and Applications 15 (2) (1994) 592-622.

[28] M. Gu, S. Eisenstat, Efficient algorithms for computing a strong rankrevealing qr factorization, SIAM J. Sci. Comput. 17 (4) (1996) 848-869.

[29] MATLAB, version 7.11.0 (R2010b), The MathWorks Inc., Natick, Massachusetts, 2010.

[30] E. Anderson, Z. Bai, C. Bischof, S. Blackford, J. Demmel, J. Dongarra, J. Du Croz, A. Greenbaum, S. Hammarling, A. McKenney, D. Sorensen, LAPACK Users' Guide, 3rd Edition, SIAM, Philadelphia, PA, 1999. 